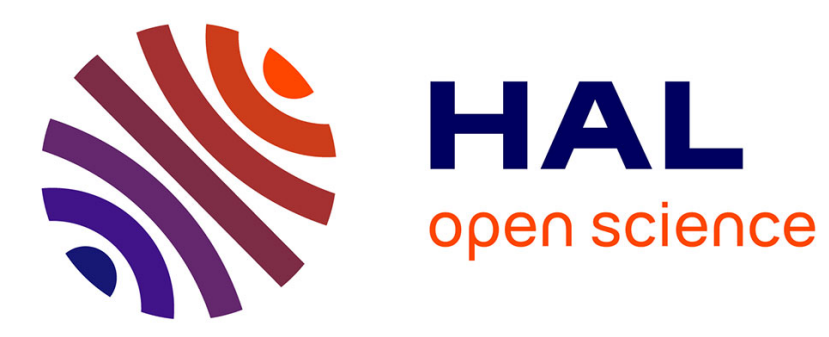

\title{
Les vulnérabilités des sociétés et des espaces urbanisés : concepts, typologie, modes d'analyse
}

Robert d'Ercole, Jean-Claude Thouret, Olivier Dollfus, Jean-Pierre Asté

\section{To cite this version:}

Robert d'Ercole, Jean-Claude Thouret, Olivier Dollfus, Jean-Pierre Asté. Les vulnérabilités des sociétés et des espaces urbanisés: concepts, typologie, modes d'analyse. Revue de Géographie Alpine / Journal of Alpine Research, 1994, Croissance urbaine et risques naturels dans les montagnes des pays en développement, 82 (4), pp.87-96. 10.3406/rga.1994.3776 . hal-01164931

\section{HAL Id: hal-01164931 \\ https://hal.science/hal-01164931}

Submitted on 18 Jun 2015

HAL is a multi-disciplinary open access archive for the deposit and dissemination of scientific research documents, whether they are published or not. The documents may come from teaching and research institutions in France or abroad, or from public or private research centers.
L'archive ouverte pluridisciplinaire HAL, est destinée au dépôt et à la diffusion de documents scientifiques de niveau recherche, publiés ou non, émanant des établissements d'enseignement et de recherche français ou étrangers, des laboratoires publics ou privés. 


\section{Robert D'Ercole \\ M. Jean-Claude Thouret \\ Olivier Dollfus \\ M. Jean-Pierre Asté}

\section{Les vulnérabilités des sociétés et des espaces urbanisés :} concepts, typologie, modes d'analyse

In: Revue de géographie alpine. 1994, Tome 82 N4. pp. 87-96.

Citer ce document / Cite this document :

D'Ercole Robert, Thouret Jean-Claude, Dollfus Olivier, Asté Jean-Pierre. Les vulnérabilités des sociétés et des espaces urbanisés : concepts, typologie, modes d'analyse. In: Revue de géographie alpine. 1994, Tome 82 №4. pp. 87-96.

doi : $10.3406 /$ rga.1994.3776

http://www.persee.fr/web/revues/home/prescript/article/rga_0035-1121_1994_num_82_4_3776 


\section{Thème II \\ Les vulnérabilités des sociétés et des espaces urbanisés : concepts, typologie, modes d'analyse}

Nous abordons ce thème à partir de la vingtaine de communications qui, au cours du colloque de Clermont-Ferrand ont traité de cette question ${ }^{1}$. Dans un premier temps, notre synthèse présente les concepts et les différentes approches de la vulnérabilité. Un regard critique est ensuite porté sur la relation entre l'évaluation et la réduction de la vulnérabilité. Enfin, les possibilités de convergence entre les différentes approches sont examinées et des pistes de recherche sont lancées.

\section{Les concepts et les approches}

\section{Le système de vulnérabilité}

La vulnérabilité apparaît dans certaines communications comme un système, articulé autour d'un grand nombre de variables, naturelles et humaines, dont la dynamique dans le temps et dans l'espace peut engendrer des situations plus ou moins dangereuses pour une société exposée. En milieu urbain, ce système trouve ses racines dans le fonctionnement même de la ville ${ }^{2}$ et/ou à l'extérieur de celle-ci (Dory). Il semble ainsi difficile de distinguer une vulnérabilité liée aux phénomènes naturels, d'une vulnérabilité, produit de phénomènes sociaux spécifiquement urbains. D'où l'expression "risque urbain " parfois préférée à celle de " risques naturels dans la ville ".

Le système de vulnérabilité se compose d'éléments vulnérables et des facteurs (Fig. 1). A partir de là, trois démarches sont proposées. Elles sont sensiblement différentes mais elles poursuivent un objectif identique, à savoir l'analyse de la vulnérabilité.

\section{Démarche qualitative portant sur les facteurs} de vulnérabilité

Cette démarche vise à cerner la vulnérabilité à travers les différents facteurs qui tendent à la faire varier. La vulnérabilité apparaît comme la propension d'une société donnée à subir des
1. 12 communications ont directement abordé cette question (cf. annexe à la fin du volumel

2. Voir l'article de November, ci-après thème 3 | 
dommages en cas de manifestation d'un phénomène naturel ou anthropique. Cette propension varie selon le poids de certains facteurs qu'il est nécessaire d'identifier et d'analyser car ils induisent un certain type de réponse de la société. Une méthode d'approche de ces facteurs débouchant sur une typologie des réponses sociales a été proposée (Thouret et D'Ercole). Dans la plupart des communications, les facteurs de vulnérabilité apparaissent pour illustrer un discours portant sur l'aléa, le risque ou les outils associés, plus rarement comme objet d'étude. Cependant, si certains facteurs apparaissent très fréquemment, d'autres sont à peine signalés. L'approche est parfois générale et concerne les P.E.D. (Garry et Veyret) ou l'Amérique Latine (Collin-Delavaud). Plus souvent, il s'agit d'études de cas localisées.

Le problème de la croissance démographique et urbaine et de son accélération revient fréquemment : à Arequipa (Pérou), Manizales (Colombic), Kingston (Jamaïque), Antananarivo (Madagascar) ou encore Katmandu (Népal). Il en va de même pour les modes d'occupation et d'utilisation du sol qui accroissent l'exposition aux menaces. Il s'agit par exemple d'occupation de zones inondables à Arequipa, à Limbé (Cameroun) ${ }^{3}$ ou à la Réunion; de zones susceptibles d'être affectées par les produits d'une éruption volcanique à Cartago (Costa-Rica) ou sur les flancs du Merapi à Java ; de secteurs urbanisés sur des failles actives comme à Chlef (Algérie). Loccupation irrationnelle de terrains à forte pente, la destabilisation de versants par déboisement ou les écoulements modifiés par l'urbanisation constituent également des facteurs de vulnérabilité à Puriscal (Costa-Rica), Pereira (Colombie), Limbé, Karmandou, etc.

Les facteurs socio-économiques de vulnérabilité sont généralement associés aux modes d'occupation du sol. La toile de fond est constituée par le processus d'urbanisation dans un contexte de crise ou de fluctuations économiques (Carreño ${ }^{4}$, Dory). Plus précisément, l'exode rural, la spéculation foncière, les pratiques clientélistes, la recherche de la proximité des centres actifs de la ville, constituent les principaux facteurs évoqués pour comprendre l'afflux de populations contraintes d'occuper des terrains à risque. C'est le cas de la plupart des grandes villes

3. Voir l'article de Zogning. ci-dessus (thème 1)

4. Voir l'article ci-dessus (thème 1) d'Amérique Latine (Collin-Delavaud). Les possibilités illusoires de tirer profit des catastrophes et des promesses de la reconstruction débouchent sur le même type de situation (exemple de la ville de Popayan en Colombie). Mais le résultat 
de ces situations est-il toujours en défaveur des groupes sociaux les plus pauvres ? Cela est communément admis, mais n'est pas toujours prouvé (Dory).

Les facteurs psycho-sociologiques de vulnérabilité, parfois traités en eux-mêmes (Colbeau-Justin), apparaissent généralement de manière ponctuelle dans les communications. Dans certains cas les gens ignorent vivre dans un secteur dangereux (Garry et Veyret), mais dans l'ensemble les risques naturels sont au moins perçus et font partie du quotidien (Collin-Delavaud). La question est donc de comprendre comment les personnes exposées perçoivent et vivent le risque. La mémoire des catastrophes qui s'atténue et se déforme avec le temps ou l'habitude de vivre des phénomènes peut-être graves mais non exceptionnels, associée à la sous-estimation de l'ampleur potentielle de certains d'entre eux, font baisser la garde. C'est entre autres le cas à Arequipa ou dans les villes antillaises. Le sentiment de sécurité provoqué par les ouvrages de protection et de manière générale par les progrès techniques ont un effet identique, par exemple à Popayan. A cela s'ajoute la peur ou les attitudes fatalistes, confortées par le discours souvent alarmiste ou sensationnaliste des médias (Bermudez). Mais les menaces naturelles ne sont pas seulement perçues et vécues d'après la représentation de leurs effets, elles entrent en effet en concurrence avec d'autres contraintes d'ordre économique et social et du coup s'en trouvent relativisées. Les risques naturels sont ainsi souvent moins obsédants que d'autres risques dits sociaux ou urbains que sont le chômage, l'insécurité, la drogue, etc. (Dory, November).

A ces facteurs psycho-sociologiques s'ajoutent des facteurs davantage liés à la culture et à l'histoire des sociétés exposées. C'est le cas de la transculturation imposée par les colonisations et l'adoption de modèles occidentaux (comme les types de constructions), peu conformes aux besoins de sécurité des P.E.D. Cusco au Pérou en fournit un exemple. Ce sont les traditions, autoconstruction, pratiques politiques laxistes, rejet dans la nature de déchets et produits encombrants, qui constituent aujourd'hui non seulement une atteinte à l'environnement mais des sources de risque (Pagney ${ }^{5}$ ). C'est également la fatalité et la résignation liées aux croyances, aux religions, aux superstitions (Colbeau-Justin, Mora et Bermudez).

En ce qui concerne les facteurs techniques de vulnérabilité, l'accent est placé sur la mauvaise qualité des constructions généra-

5. Voir Particle ci-dessus (thème 1 ) 
lement inadaptées au milieu (à Cusco, par exemple) ou aux contraintes sismiques (à Chlef, à Limbé). Les inondations sont favorisées par une incapacité technique de maîtrise des eaux, le sous-dimensionnement et l'absence d'entretien des ouvrages. Les systèmes techniques de construction des routes s'avèrent souvent inadéquats et facilitent le développement de mouvements de terrain (à Katmandu, à Puriscal au Costa Rica). Les ouvrages de protection face à certains effets des éruptions volcaniques sont trop rudimentaires (exemple de Cartago au Costa Rica ou aux Philippines dans les régions exposées à l'activité du volcan Pinatubo).

Alors que les facteurs techniques touchent essentiellement à la prévention, les facteurs fonctionnels concernent la gestion des crises et plus précisément la qualité opérationnelle des organisations et des moyens techniques et humains mis en oeuvre. D'après J.-P. Gout, dans la mesure où la maîtrise de l'occupation du sol est très difficile, il est nécessaire d'axer les efforts vers la prévision temporelle et la mise en place de structures locales de sécurité civile (" le maillon manquant " entre les structures régionales et la population). Le problème de la prévision, de la surveillance et de l'alerte est également signalé à propos de crues de rivières à très faible temps de réponse (exemple des Antilles) ou de lahars à Quito. Par ailleurs, la communication difficile entre les divers acteurs (scientifiques, décideurs, journalistes et population) constitue un obstacle majeur (Velasquez et al.).

Avec les facteurs institutionnels et politico-administratifs de vulnérabilité, ce sont les rouages du système de gestion des risques qui sont à appréhender en considérant notamment les échelles et domaines de compétence des organismes concernés et la qualité des relais aux niveaux national, régional et local, afin de mettre en évidence les dysfonctionnements (Thouret et D'Ercole). Il s'agit aussi de faire apparaître, à l'aide d'indicateurs, les facteurs technico-administratifs ou les enjeux politiques et stratégiques susceptibles de freiner une gestion rationnelle des risques. A défaut d'une véritable analyse, ce sont les insuffisances en la matière qui ont été soulignées. On signale l'absence de programmes de prévention, de préparation ou de manière plus générale de réflexion sur les risques dans le cadre de la planification urbaine. Les pratiques politiques laxistes, les récupérations politiciennes, les opérations préventives bloquées par la pression sociale, l'obligation de valider des situations de 
fait dangereuses, sont parfois évoquées (à Antananarivo, à la Réunion, ou de manière générale en Amérique Latine). La question de la législation et des réglementations est à peine discutée, si ce n'est pour mettre en évidence leur inexistence ou pour éclairer un fait fort juste : les outils juridiques mis en place par les P.E.D. sont parfois plus nombreux que ce que l'on pense habituellement, en revanche ils pêchent par leur inefficacité (Collin-Delavaud).

Quelques autres facteurs de vulnérabilité ont été signalés, notamment la question des assurances dont la couverture est très faible dans les P.E.D. (Ledoux ${ }^{6}$ ). Il s'agit aussi des facteurs conjoncturels, s'opposant à l'ensemble des facteurs signalés cidessus que l'on peut considérer comme structurels (Thouret et D'Ercole) : lieu et moment précis de l'impact, dysfonctionnements urbains, fonctionnels et techniques imprévisibles, etc.

\section{Démarche semi-quantitative intégrant à la fois les facteurs de vulnérabilité et les éléments vulnérables}

Cette deuxième démarche découle de la précédente. La vulnérabilité est toujours considérée comme une propension à subir des dommages, mais la société n'est plus appréciée de manière globale ou suivant les thématiques résumées ci-dessus. La démarche va plus loin car, semi-quantitative, elle débouche sur une hiérarchisation sociale et/ou spatiale des éléments exposés (les habitants d'une ville, leurs biens ou leurs activités). L'un de ses objectifs est l'élaboration de cartes de vulnérabilité. Deux communications illustrent cette démarche.

L'étude de Lavigne et Thouret ${ }^{7}$ porte sur les régions exposées aux lahars du volcan Merapi (Java, Indonésie). Au stade méthodologique, elle propose d'évaluer la vulnérabilité des populations, du patrimoine et de l'économie locale à l'intérieur de zones obtenues par un microzonage cartographique des lahars. La méthode de A.C. Chardon ${ }^{8}$ concernant la vulnérabilité de la ville de Manizales (Colombie) face à un ensemble de risques naturels est proche de la précédente dans l'esprit, mais sensiblement différente sur le plan technique. La hiérarchisation spatiale de la vulnérabilité et la cartographie des zones vulnérables sont issues du croisement d'une quinzaine de facteurs naturels, techniques et sociaux de vulnérabilité choisis comme indicateurs pertinents.
6. Voir l'article ci-après (thème 3)

7. Voir l'article ci-après (thème 3)

8. Voir l'article ci-après (thème 2) 


\section{Démarche quantitative portant sur les éléments vulnérables}

Alors que les deux démarches précédentes sont liées, la troisième s'est, jusqu'à présent, totalement démarquée des premières. Elle se fonde essentiellement sur l'élément vulnérable à partir duquel il s'agit de mesurer les conséquences, en cas de survenue d'un phénomène générateur de dommages. La vulnérabilité est ici conçue comme le pourcentage (ou une valeur allant de 0 à 1) de ce qui peut être perdu en cas de sinistre. Cette démarche est employée depuis longtemps, notamment par certains économistes dont le but est d'aider les décideurs à formuler et à chiffrer leurs politiques de prévention. Suivant des techniques d'évaluation parfois complexes, elle vise à mettre en balance les répercussions économiques des dégâts occasionnés par une catastrophe potentielle et les coûts entraînés par la réalisation de mesures visant à en limiter les effets. Il s'agit là d'analyses de vulnérabilité stricto sensu, associées à des analyses coûts-bénéfices.

Les travaux de $\mathrm{S}$. Mora, à propos de la ville de Cartago au Costa Rica, reposent sur cette logique. Les dommages humains et économiques liés à des laves torrentielles du rio Reventado issues du volcan Irazú sont évalués selon trois scénarios possibles (faible, moyenne ou grande extension des lahars). Ce type d'étude demeure cependant indicatif, en raison de biais volontaires mais importants dans l'estimation des paramètres et des effets des phénomènes destructeurs et dans l'évaluation des modes d'endommagement des éléments exposés. Suivant ce constat et à propos de mouvements de terrain, Leone et al. proposent une méthode de constat d'endommagement, dont l'un des objectifs est de fournir une évaluation précise des dommages potentiels, et par conséquent, de la vulnérabilité.

\section{De l'évaluation à la réduction de la vulnérabilité}

\section{De la vulnérabilité à la gestion des risques}

Il est clair que quelle que soit la manière d'évaluer ou de mesurer la vulnérabilité, le but ultime est de tenter de la réduire. Il s'agit alors de porter un regard critique sur les liens existant entre les approches à l'amont et les applications à l'aval. Pour simplifier, on observe au moins trois types de situations : 
- Certains considèrent et présentent la vulnérabilité comme un système où éléments vulnérables et facteurs de vulnérabilité sont en interaction, mais ne débouchent pas sur des propositions concrètes de réduction.

- D'autres évoquent ou analysent certains facteurs de vulnérabilité et proposent parfois des solutions directement liées aux problèmes évoqués. Cependant, si ces solutions paraissent pertinentes en elles-mêmes, elles ne tiennent généralement pas compte du contexte et des contraintes qui rendent leur utilisation ou leur application difficile, voire impossible.

- D'autres encore se situent à l'aval et proposent des outils d'aide à la décision ou de gestion des risques. Toutefois, ils sont parfois déconnectés de la diversité des problèmes amont, car ils ne considèrent que certains aspects de la question, voire un seul.

Ces différentes approches sont à l'évidence extrêmement utiles et novatrices pour la plupart, mais le lien entre la phase d'évaluation de la vulnérabilité et celle qui, à travers méthodes et outils divers, vise à la réduire, pose problème. Cette vision simplificatrice de la réalité doit cependant être nuancée, car plusieurs communications tentent de bâtir des relations étroites entre ces deux phases. Il s'agit par exemple des études portant sur le transfert de méthodes occidentales dans les P.E.D. (Garry et Veyret, Ledoux, Rosenfeld et Pearson). D'autres contributions se préoccupent peut-être davantage de la question des relations, mais en sont encore au stade méthodologique (Chardon, Leone et al., Prieto et Cardona, Thouret et D'Ercole...). Il s'agit donc là d'un axe de recherche important à développer.

\section{De la gestion des crises à l'évaluation de la vulnérabilité}

Une démarche inverse de la précédente consiste à partir de la crise et de ses conséquences et de considérer les actions de réduction de la vulnérabilité entreprises dans le cadre d'une reconstruction et d'une réhabilitation consécutives à un désastre. La vulnérabilité des collectivités affectées, affichée avant la catastrophe, peut varier très sensiblement suivant la manière dont les périodes postérieures aux crises sont gérées. Elle peut être atténuée ou au contraire amplifiée. Elle peut également changer de nature. L'intérêt de la démarche est présentée à propos de la ville de Popayan (D'Ercole). 


\section{Vers une convergence des différentes approches et perspectives}

\section{Vers une convergence par l'analyse de système}

Les communications traitant de la vulnérabilité des espaces urbanisés et les débats engagés ont fourni des éléments de réponse à trois grosses questions préalablement posées :

- le concept de vulnérabilité a-t-il la même signification pour tous?

- comment appréhender la vulnérabilité compte-tenu de la pluralité des variables qui la composent, notamment en milieu urbain et dans les P.E.D. ? La vulnérabilité s'inscrit-elle alors dans une analyse de système?

- comment éviter l'éclatement des approches et tendre vers une réflexion commune permettant en particulier de bâtir un lien plus étroit entre l'évaluation et la réduction de la vulnérabilité ?

La réponse à la première question est claire. Le concept de vulnérabilité n'a pas la même signification pour tous et cela a été démontré. A l'approche classique de la vulnérabilité qui mesure un endommagement potentiel des biens et des personnes et ses répercussions sur l'environnement économique, semble s'opposer celle qui considère la vulnérabilité des sociétés à travers leur capacité de réponse à des crises potentielles. Cette capacité est elle-même liée à un ensemble de facteurs structurels et contingents qui peuvent être analysés séparément, mais dont les interrelations s'avèrent complexes. C'est pourquoi, toute analyse de vulnérabilité, surtout lorsqu'il s'agit du monde urbain qui tend à multiplier et diversifier les facteurs de vulnérabilité, peut difficilement s'affranchir d'une démarche systémique.

Suivant cette démarche, il ne peut être concevable de dissocier chacun des éléments du système (éléments vulnérables et/ou facteurs) si ce n'est de manière artificielle, à des fins d'analyse. Mais l'analyse doit être considérée comme un préalable à une appréciation globale des conditions de vulnérabilité, elle-même précédant toute entreprise efficace de réduction. Par exemple, agir sur des aspects purement techniques, raison apparente de la vulnérabilité, risque de n'avoir qu'un effet limité si des facteurs économiques sont en cause, et ces derniers peuvent s'avérer secondaires si les valeurs sociales constituent le problème de fond. Seule une approche systémique peut ainsi permettre 


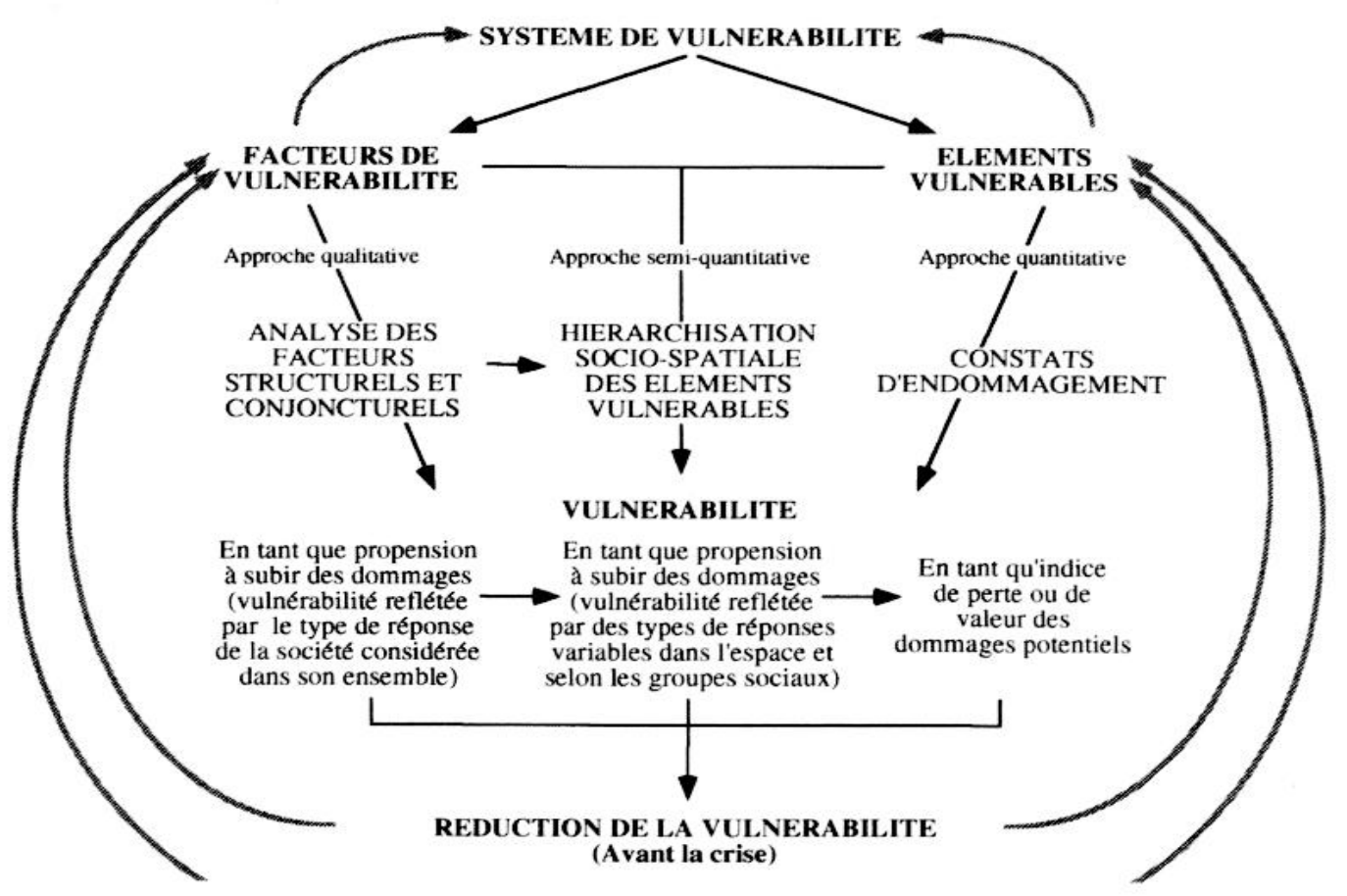

GESTION POST-CRISE

d'identifier les véritables cibles sur lesquelles on peut tenter d'agir. Des travaux de recherches doivent se développer dans cette direction.

La synthèse des diverses contributions ayant abordé la question de la vulnérabilité (Fig. 1), permet de concevoir l'intérêt de l'approche systémique et de mettre en évidence des liens entre des approches qui, loin de s'opposer, s'avèrent en fait complémentaires. C'est ainsi qu'un constat d'endommagement apparaît insuffisant sans une bonne appréciation de la propension à subir des dommages. Mais l'inverse est aussi vrai. De plus, il existe des liens étroits entre cette propension qui répond à un certain nombre de facteurs de vulnérabilité en interaction, et un indice de perte issu de considérations purement techniques. C'est aussi sur ces liens que des efforts de recherche doivent être entrepris pour une plus juste appréciation de la vulnérabilité. Celle-ci permettrait de concevoir des outils plus adaptés aux différentes situations en agissant sur les éléments vulnérables et/ou sur les facteurs, ou, en d'autres termes, sur le système lui-même.
Synthèse des différentes approches de la vulnérabilité ef relations 


\section{La question de l'élément vulnérable}

Une dernière question, entre autres, reste en suspens : celle de l'élément vulnérable. Quelle que soit l'approche de la vulnérabilité proposée lors du colloque, les éléments vulnérables se limitent essentiellement aux personnes, aux biens et quelquefois aux enjeux économiques. Il s'agit là d'un choix réducteur de la réalité, commode dans la mesure où il s'appuie sur des données quantitatives. Cependant, les éléments vulnérables (valeurs ou enjeux) peuvent être également d'ordre social, culturel, esthétique ou encore environnemental. Les enjeux sont aussi d'ordre géographique lorsque les modalités de développement d'une ville ou les hiérarchies urbaines dans une région donnée peuvent être remises en question en cas de catastrophe. Ces éléments sont évidemment moins commodes à évaluer, car moins quantifiables, mais ils ne demeurent pas moins importants à considérer pour une approche globale de la vulnérabilité. Là encore des pistes de recherche sont ouvertes.

En conclusion, la table-ronde consacrée aux vulnérabilités des sociétés et des espaces urbanisés a mis en évidence des divergences importantes à propos des concepts et des approches de la vulnérabilité. En dépit de la qualité des travaux réalisés, ces divergences nuisent à l'efficacité d'une recherche dont le but final est la réduction de la vulnérabilité. Principalement liées au cloisonnement actuel de la recherche dans ce domaine (cloisonnement pluridisciplinaire ou au sein d'une discipline comme la géographie), elles sont en fait plus apparentes que réelles. En effet, les différentes approches peuvent converger dans le cadre d'une analyse de système qui semble particulièrement adaptée au milieu urbain, où les variables à prendre en compte sont nombreuses, variées et complexes dans leur interaction. Des pistes nouvelles de recherche sont ainsi lancées. 